\title{
Les relatives appositives entre intégration syntaxique et intégration discursive
}

\author{
Lytvynova, Maryna, \& Dao, Huy Linh \\ LATTICE, Université Sorbonne Nouvelle Paris 3 \& LACITO, Université Sorbonne Nouvelle Paris 3 \\ lytvynova maryna@yahoo.fr \& dao.huy.linh@gmail.com
}

L'objectif du présent travail est de mettre en lumière certaines propriétés discursives des relatives appositives du français et, par la même occasion, de participer au débat sur le statut dont elles jouissent au niveau du discours. Plus spécifiquement, en prenant comme point de départ la discussion antérieure (cf. Brandt, 1990 ; Combettes, 1992 ; Lambrecht, 1998 ; Holler, 2005b ; Loock, 2007) portant sur la distinction fonctionnelle entre les relatives narratives (1) et les relatives descriptives (2), nous suggérons que, contrairement à ce que postulent les travaux précédents, ni les premières, ni les secondes ne peuvent être assimilées à des unités autonomes du discours, l'hypothèse que nous étayons avec des données expérimentales.

\section{Introduction}

Il est bien connu depuis Emonds (1979) que les relatives appositives se distinguent assez nettement des relatives restrictives dans la mesure où, contrairement à ces dernières, elles présentent plusieurs propriétés typiquement associées aux propositions indépendantes, comme, par exemple, le fait qu'elles échappent régulièrement à la portée des quantificateurs présents dans leur matrice; qu'elles se comportent comme des unités prosodiques séparées; et qu'elles acceptent la présence d'adverbiaux d'énonciation (eg. franchement), autrement dit, elles semblent fonctionner comme des actes de langage indépendants ( $c f$. Krifka 2009).

Ces particularités des relatives appositives vis-à-vis des subordonnées standards ont amené plusieurs analyses précédentes à les traiter comme étant autonomes de leurs matrices tant sur le plan grammatical que sur le plan pragmatique (cf. Peterson, 2003 ; Holler, 2005b).

A cela, la littérature sur le discours (Brandt, 1990 ; Combettes, 1992 ; Lambrecht, 1998 ; Loock, 2007 , inter alia) ajoute une distinction supplémentaire entre les relatives appositives narratives et les relatives appositives non narratives (ci-après descriptives), illustrées dans (1) et (2) respectivement:

(1) Louise fit signe au garçon, qui lui apporta l'addition.

(2) Louise fit signe au garçon, qui feuilletait un journal.

La spécificité des relatives narratives par rapport à leurs homologues descriptives, celle qui est à l'origine du statut «privilégié » qu'elles se voient attribuer dans les travaux antérieurs, réside dans le fait qu'elles font progresser la chronologie du récit. En effet, contrairement à la relative de (2), qui dénote un état de choses contingent à celui exprimé par sa matrice, les deux étant repérés par rapport au même temps de référence, la situation décrite par la relative de (1) est perçue comme étant postérieure à (et, éventuellement, déclenchée par) l'évènement mis en place par sa principale. Or, cette propriété, qui joue un rôle crucial dans la détermination de ce qui constitue la ligne principale d'un texte (cf. Reinhart, 1981 ; Thompson, 1987), i.e. son avant-plan ou premier plan (ang. foreground), est considérée comme étant atypique pour les propositions subordonnées, l'idée sous-jacente étant que le statut syntaxique d'une proposition prédétermine en quelque sorte son interprétation discursive : les indépendantes apportant des informations décisives pour le développement du discours (i.e. avant-plan) et les subordonnées des informations sans incidence majeure sur son avancée (i.e. arrière-plan) ${ }^{1}$ :

«Par leur fonction au niveau du discours, par leur rôle informationnel, les subordonnées correspondent [...], dans la plupart des cas, à un apport qui ne relève pas 
du premier plan : descriptions, commentaires, explications avec relatives, les causales, les consécutives, etc., alors que les propositions principales maintiennent le fil conducteur du premier plan » (Combettes, $1992: 124)$.

\section{Traitements antérieurs}

Brandt (1990) exprime la distinction entre les relatives narratives et descriptives à travers la corrélation entre le potentiel communicatif d'une proposition et le type d'informations qu'elle est susceptible de véhiculer une fois intégrée dans le discours. Ainsi, les subordonnées seraient des structures à potentiel communicatif bas, réservées à apporter des informations mineures (i.e. d'arrière-plan), et les indépendantes des structures à potentiel communicatif élevé, exprimant des informations majeures pour le discours (i.e. d'avant-plan). L'assignation d'un potentiel communicatif étant définie comme marquage linguistique du degré d'«importance» qu'un locuteur attribue au contenu qu'il énonce, deux unités informatives $\alpha$ et $\beta$ reçoivent des statuts discursifs différents, majeur et mineur respectivement, s'ils sont réunis dans une phrase complexe où le constituant dénotant $\beta$ dépend de celui dénotant $\alpha$. Inversement, $\alpha$ et $\beta$ reçoivent le même statut majeur, s'ils sont présentés séquentiellement. Crucialement, les phrases complexes comportant une relative narrative sont traitées ici de la même façon qu'une suite de propositions indépendantes juxtaposées ou coordonnées au sein d'un discours : les relatives narratives se voient assigner le même potentiel communicatif élevé que leurs matrices, les deux propositions véhiculant des informations majeures pour leur discours d'accueil.

Il convient de noter que, dans cette approche, la notion d'informations majeures ne correspond pas exactement à celle d'avant-plan issue des travaux sur les textes narratifs (cf. supra), la définition des deux notions étant fondée sur des critères différents. En effet, pour arriver à la conclusion sur le statut majeur des relatives narratives, Brandt ne s'appuie pas tellement sur l'aptitude de ces propositions à faire progresser le temps du récit mais sur deux autres propriétés que, selon elle, possèdent les relatives narratives et qui font défaut aux relatives descriptives. Notamment, les premières, contrairement aux secondes, se prêtent à la reformulation par une proposition coordonnée (3) et à leur utilisation comme le premier élément dans la relation de concession induite par dennoch 'toutefois, pourtant' (4) :

a. Sie gab das Buch Emil, der es dann zur Bibliothek brachte ${ }^{2}$.

Elle donna le livre Emil qui le alors à-la bibliothèque porta

'Elle donna le livre à Emil, qui le porta ensuite à la bibliothèque'

b. Sie gab das Buch Emil und er brachte es dann zur Bibliothek.

Elle donna le livre Emil et il porta le alors à-la bibliothèque

'Elle donna le livre à Emil et il le porta ensuite à la bibliothèque'

(4) Sie machten dann ihr Experiment, das auch gelang. Dennoch wurde die Untersuchung abgebrochen

Ils menèrent alors leur expérience qui PART réussit. Toutefois fut la recherche abandonnée

'Ils menèrent leur expérience, qui réussit. Toutefois, la recherche fut abandonnée'

Holler (2005b) adhère à l'hypothèse de Brandt mais suggère que la notion d'assignation de potentiel communicatif devrait être définie sur des bases strictement discursives. Selon cette auteure, toutes les relatives appositives, qu'elles soient narratives ou descriptives, sont autonomes de leurs matrices tant syntactico-sémantiquement qu'informationnellement ${ }^{3}$ et, par conséquence, leur interprétation discursive ne peut pas être fonction de leur statut grammatical. En adoptant le cadre de la SDRT (Asher, 1993), elle postule qu'assigner un potentiel communicatif à une unité informative indépendante revient à déterminer son statut rhétorique vis-à-vis du contexte linguistique antérieur. Plus concrètement, un constituant $\alpha$ aura un potentiel communicatif élevé s'il est attaché à ce qui précède par une relation discursive de coordination, et il aura un potentiel communicatif bas s'il est rhétoriquement subordonné au discours précédent. Partant de là, dans cette approche, la relative de (5) possède un potentiel communicatif élevé parce qu'elle est coordonnée rhétoriquement à sa matrice, les deux propositions entretenant entre elles 
une relation de Narration, relation qui n'induit pas de rapports de dominance entre les constituants qu'elle relie :

(5) Oskar traf einen Bauern, den er dann nach dem Weg fragte.

Oscar rencontra un fermier auquel il alors pour le chemin demanda

'Oscar rencontra un fermier, auquel il demanda alors le chemin'

Pour ce qui est de la relative descriptive de (6), d'après Holler, elle serait attachée à sa principale par la relation d'Elaboration. Or, cette relation impliquant un rapport de dominance asymétrique entre les constituants auxquels elle s'applique, les relatives descriptives seraient donc subordonnées rhétoriquement à leurs matrices, d'où le potentiel communicatif bas qu'elles reçoivent :

(6) Oskar traf einen Bauern, der übrigens einen Strohhut trug.

Oscar rencontra un fermier qui d'ailleurs un de-paille-chapeau portait

'Oscar rencontra un fermier, qui d'ailleurs portait un chapeau de paille'

Cette manière de traiter l'interprétation pragmatique des relatives appositives présente plusieurs avantages. Premièrement, elle donne une explication théorique indépendante à la notion d'assignation de potentiel communicatif, qui reste assez vague dans le travail de Brandt (1990) et donc difficilement exploitable. Deuxièmement, elle permet de contourner les difficultés auxquelles se heurtent inévitablement les analyses qui, pour rendre compte des différences du fonctionnement discursif des relatives narratives et descriptives, postulent leur non-équivalence syntaxique (cf. Lambrecht, 1998), idée qui manque crucialement de bases empiriques dans la mesure où les deux types de relatives semblent avoir exactement les mêmes propriétés grammaticales ${ }^{5}$.

Toutefois, l'analyse d'Holler n'est pas sans soulever des questions. Théoriquement, il est discutable que la relative de (6), répétée ci-dessous dans (8), entretienne avec sa matrice une relation d'Elaboration et est, par conséquent, subordonnée rhétoriquement à celle-ci. D’après Asher \& Lascarides (1991), $\beta$ élabore $\alpha$ si l'état de choses décrit par $\beta$ contribue à la réalisation de l'état de choses décrit par $\alpha$. Cela est illustré dans (7), où l'énoncé (b) élabore l'énoncé (a) dans la mesure où la situation qu'il décrit est interprétée comme une partie constitutive de l'évènement global mentionné par $\alpha$, i.e. le nettoyage de l'appartement :

a. Marie a nettoyé l'appartement.

b. D'abord, elle a épousseté les meubles. Ensuite, elle ...

(8) a. Oscar rentra un fermier,

b. qui portait un chapeau de paille.

Il est évident que (7) et (8) n'ont pas les mêmes propriétés. Alors que le temps de référence associé à l'état de choses évoqué par (8b) inclut celui par rapport auquel est repéré l'évènement exprimé par (8a), dans (7), c'est le temps de référence de (7b) qui se trouve inclus dans celui de (7a). Il en va de même pour l'interprétation discursive des deux séquences. L'énoncé (7b) spécifie l'une des étapes de (7a). Dans (8), en revanche, l'état de choses dénoté par $(8 b)$ n'a aucune incidence sur l'évènement de (8a), les deux étant perçus comme indépendants l'un de l'autre. La relation rhétorique qui, d'après nous, saisit plus fidèlement le lien entre $(8 a)$ et $(8 b)$ est celle d'Arrière-plan, définie et exemplifiée dans $(9)$ :

(9) Arrière-plan $(\alpha, \beta)$ : si la situation décrite par $\beta$ exprime des circonstances dans lesquelles la situation décrite par $\alpha$ a eu lieu.

a. Marc sortit dans la cours.

b. Elle était vide.

S'il est vrai que la relative de (8) sert d'arrière-plan à l'évènement décrit par sa matrice, alors la corrélation entre le potentiel communicatif d'une unité informative (bas $v s$ élevé) et son statut rhétorique vis-à-vis du discours antérieur (subordonné vs coordonné) postulée par Holler ne peut plus être maintenue dans le cadre théorique qu'elle retient du fait que la SDRT classe Arrière-plan parmi les relations discursives de coordination (cf. Asher \& Lascarides, 1996). 
Un autre problème théorique que pose l'analyse d'Holler concerne le lien qu'elle établit entre l'inaptitude des relatives descriptives à servir d'argument à dennoch 'toutefois' et leur statut de subordonnées discursives. En effet, ce que de façon plus générale, révèle le test de dennoch c'est que les relatives descriptives, à elles seules, sont incapables d'entretenir une relation rhétorique avec l'énoncé qui les suit immédiatement. Or, dans la SDRT, le fait qu'un constituant $\beta$ soit subordonné à $\alpha$ n'implique aucunement qu'un constituant subséquent à $\beta-\varphi-$ ne puisse pas être attaché rhétoriquement à $\beta$. Le statut de $\beta$ vis-àvis de $\alpha$ (i.e. subordonné ou coordonné) peut affecter l'accessibilité de $\alpha$ pour le discours postérieur, mais $\beta$ reste toujours un site d'attachement possible pour $\varphi$ (cf. Asher \& Vieu, 2005). Cela est illustré dans (10), qui montre que la relation d'Elaboration par laquelle (b) est lié à (a), i.e. la subordination rhétorique de (b) à (a), n'empêche pas le constituant (c) d'entrer en relation de Narration avec (b) :

(10) a. Pierre a passé une soirée très agréable.

b. Il a dîné avec ses amis dans un restaurant japonais.

c. Ensuite, il est allé au cinéma.

L'analyse d'Holler n'est pas non plus entièrement satisfaisante du point de vue empirique. Premièrement, l'affirmation même que seules les relatives narratives réussissent le test de coordination et celui de dennoch et sont donc les seules à pouvoir apporter des informations majeures pour le discours semble être erronée, du moins en ce qui concerne les relatives du français. Ainsi, même s'il est vrai que hors contexte un énoncé comme (11) semble peu naturel, en présence d'un contexte approprié le contenu exprimable par une relative descriptive peut être formulé à l'aide d'une proposition coordonnée (12) et il peut aussi servir de « cible » à toutefois (13) :

(11) ?? Oscar croisa une fermière et elle portait un chapeau de paille.

(12) Contexte : Récemment, Oscar a visité une région dont les habitants étaient connus pour leurs goûts vestimentaires très extravagants.

A : Alors, qui as-tu rencontré là-bas et comment ces gens étaient-ils habillés ?

B : J'ai croisé une fermière, qui portait un chapeau de paille.

B' : J'ai croisé une fermière et elle portait un chapeau de paille.

(13) Marie tendit le sac couvert de boue et de poussière à Marc, qui était vêtu de blanc des pieds à la tête. Toutefois, il prit le sac et le serra contre sa poitrine.

Le fait que les relatives descriptives soient susceptibles de recevoir une lecture d'avant-plan est confirmé également par d'autres phénomènes. Ainsi, elles peuvent être validées ou invalidées directement (14), i.e. par oui, c'est vrai ou non, c'est faux, l'idée sous-jacente étant que le contenu d'avant-plan est la partie de l'énoncé que le locuteur propose en tant que mise à jour possible du contexte discursif et que, comme toute proposition, celle de mise à jour du contexte doit pouvoir faire l'objet d'une négociation explicite de la part des interlocuteurs (Simons \& al., 2010) :

(14) A : Louise a fait signe au garçon, qui feuilletait un journal.

B : Non, c'est faux (il somnolait au comptoir).

Deuxièmement, même si l'assignation d'une relation de coordination d'Arrière-plan aux relatives descriptives peut expliquer les données présentées dans (12) - (14), l'idée de dériver l'interprétation pragmatique des relatives appositives (d'avant-plan ou d'arrière-plan) du type de relations rhétoriques qu'elles entretiennent avec leur principale échoue à rendre compte de l'instabilité du statut pragmatique de ces propositions. Ainsi, dans (15), qui est sorti est une relative narrative, qui est, par conséquent, coordonnée rhétoriquement à sa matrice Marie a dit quelques mots à Jean :

(15) $\mathrm{A}:$ [Marie a dit quelques mots à Jean $]_{\mathrm{P} 1}$, [qui est sorti $]_{\mathrm{P} 2}$.

B : C'est vrai ?

$\mathrm{C}$ : Oui, (?? Jean est sorti. / ${ }^{\mathrm{OK}}$ Marie a dit quelques mots à Jean.)

Toutefois, la relative reçoit une lecture d'arrière-plan. En effet, étant donné la nature «négociable » de tout contenu d'avant-plan, i.e. son aptitude à faire l'objet d'une discussion explicite de la part des 
interlocuteurs, le fait que la question de B s'interprète plus naturellement comme demandant une confirmation de la vérité du contenu de la matrice suggère que c'est cette dernière qui obtient le statut de porteuse d'informations majeures et cela malgré la coordination rhétorique de la relative qui suit.

Et, enfin, il nous semble nécessaire de souligner que la variabilité d'interprétation pragmatique des relatives appositives n'est pas attestée dans les cas où le même contenu est exprimé par des propositions indépendantes. Ceci invite à se demander si effectivement, comme le prétend Holler, les appositives sont assimilables aux prédications autonomes du point de vue discursif. Ainsi, comme on peut le constater en comparant (15) et (16), l'expansion de la relative, qui a pour effet de rendre son contenu plus informatif que celui de sa proposition enchâssante, permet à la relative d'obtenir une interprétation majeure, corrélation qui n'existe pas dans (17) et (18). Cela signifie qu'alors que les propositions indépendantes sont toujours appréhendées comme contenant des informations d'avant-plan, la lecture pragmatique des relatives appositives dépend du degré d'informativité de leur matrice : plus celle-ci est légère du point de vue informatif et ancrée dans le contexte antérieur ou, formulé autrement, plus la relative est riche en informations et plus l'évènement qu'elle dénote est « hors du commun », plus elle a de chances d'obtenir le statut de composante d'avant-plan :

(16) A : [Marie a dit quelques mots à Jean $]_{\mathrm{P} 1}$, [qui est sorti en lui jetant un regard plein de haine et en claquant bruyamment la porte $]_{\mathrm{P} 2}$.

$\mathrm{B}:$ C'est vrai ?

$\mathrm{C}$ : Oui, $\left({ }^{\mathrm{OK}} \mathrm{Jean}\right.$ est sorti comme un fou. / ?? Marie a dit quelques mots à Jean.)

(17) $\mathrm{A}:[\text { Marie a dit quelques mots à Jean }]_{\mathrm{P} 1}$. [Il est sorti $]_{\mathrm{P} 2}$.

$\mathrm{B}:$ C'est vrai ?

$\mathrm{C}$ : Oui, $\left({ }^{\mathrm{OK}}\right.$ Jean est sorti. / ${ }^{\#}$ Marie a dit quelques mots à Jean $)$

(18) A : [Marie a dit quelques mots à Jean $]_{\mathrm{P} 1}$. [Il est sorti en lui jetant un regard plein de haine et en claquant bruyamment la porte $]_{\mathrm{P} 2}$.

B : C'est vrai ?

$\mathrm{C}$ : Oui, $\left({ }^{\mathrm{OK}} \mathrm{Jean}\right.$ est sorti comme un fou. / ${ }^{\#}$ Marie a dit quelques mots à Jean.)

Sur la base de ces observations, nous proposons dans la suite de ce travail d'examiner plus en détails l'hypothèse selon laquelle les relatives appositives fonctionneraient discursivement comme des unités informatives indépendantes et, en tant que telles, seraient sujettes aux mêmes règles et contraintes discursives que les unités réalisées par des prédications autonomes. Plus spécifiquement, nous proposons de voir comment les relatives appositives - narratives et descriptives - affectent le degré d'accessibilité cognitive des entités figurant dans leurs discours d'accueil et si, de ce point de vue, elles sont comparables à leurs homologues indépendantes. Pour ce faire, nous nous appuyons sur le cadre de la théorie du centrage d'attention (Walker \& al., 1998) - théorie de la cohérence référentielle du discours que nous complétons avec des idées provenant de la SDRT.

\section{Accessibilité référentielle : Théorie du centrage d'attention et SDRT}

L'objectif principal de la théorie du centrage d'attention est de rendre compte de la dimension référentielle de la cohérence textuelle et, plus concrètement, de l'un de ses principes directeurs, selon lequel un segment discursif ${ }^{6} \mathrm{~S}$ constitué de deux énoncés indépendants $\mathrm{E}_{1}$ et $\mathrm{E}_{2}$ est perçu comme plus cohérent (i) si $\mathrm{E}_{1}$ et $\mathrm{E}_{2}$ sont marqués par la continuité topicale ; et (ii) si l'expression linguistique par laquelle une entité $e$ évoquée dans $\mathrm{E}_{1}$ est réintroduite dans $\mathrm{E}_{2}$ correspond au degré d'accessibilité cognitive que $e$ reçoit dans $\mathrm{E}_{1}$, l'idée sous-jacente étant que le référent le plus saillant (ou accessible) d'E $E_{1}$, s'il est mentionné dans $E_{2}$, doit être réalisé dans $E_{2}$ par une forme linguistique maximalement réduite prosodiquement et sémantiquement, i.e. être cliticisé dans des langues comme le français (cf. Ariel, 1990). Etant donné que l'objectif principal de ce travail consiste à étudier si les relatives appositives et leurs homologues indépendantes affectent de la même manière l'aptitude des référents évoqués dans leur segment discursif d'accueil à servir d'antécédent à une proforme clitique figurant dans 
le discours subséquent, dans la suite, nous ne commenterons que le deuxième volet de ce principe de cohérence textuelle.

Ainsi, dans la théorie du centrage d'attention l'interprétation d'un discours est assimilé au processus de mises à jour systématiques de sa composante dynamique libellée ici focus d'attention local (ang. local (attentional) focus). A chaque étape de l'avancement du discours, le focus d'attention local véhicule deux types d'informations. D'une part, il fournit une liste de ses centres anticipateurs (Ca) (ang. forwardlooking centers), i.e. entités qui, à des degrés de probabilité variés, peuvent devenir le centre rétroactif (Cr) (ang. backward looking center) de l'énoncé suivant, autrement dit, être cliticisés dans l'énoncé suivant, le terme de Cr renvoyant à « l'objet de discours psychologiquement le plus [proéminent] à la fois pour l'énonciateur et pour l'allocutaire au moment où l'expression qui le réalise est employée [...]» (Cornish, $2000: 4$ ). Et, d'autre part, il instruit les interprétants sur la façon dont ces entités sont classées 'ranked' du point de vue de leur saillance relative. L'entité réalisée comme sujet grammatical ${ }^{7}$ de l'énoncé est classée première sur cette échelle saillantielle : elle est le centre préféré $(\mathrm{Cp})$ (ang. preferred center) de l'énoncé, dont on prédit qu'elle deviendra le $\mathrm{Cr}$ du discours subséquent. L'énoncé $\mathrm{E}_{2}$ apparaissant dans la suite immédiate d'un énoncé $\mathrm{E}_{1}$ sera donc perçu comme plus cohérent si le $\mathrm{Cr}$ d' $\mathrm{E}_{2}$ est identique au Cp d' $\mathrm{E}_{1}$. Cela est illustré dans (19) :

(19) a. Susan $_{1}$ a offert un hamster à Betsy .

b. Elle lui $_{2}$ a rappelé que les hamsters étaient assez sauvages.

b'. ? Susan lui a rappelé que les hamsters étaient assez sauvages

Le focus d'attention résultant du traitement de (19a) comprend trois Ca-SUSAN ${ }^{8}$, BETSY et HAMSTER dont SUSAN - sujet syntaxique de (19a) - est le Cp. Autrement dit, à partir de (19a), on prédit que si ce référent est évoqué dans l'énoncé subséquent, il y sera cliticisé, i.e. il sera le $\mathrm{Cr}$ de cet énoncé. Cela explique pourquoi continuer sur (19a) par (19b) produit une séquence plus satisfaisante que lorsque (19a) est suivi de (19b') : alors que dans (19b), l'expression qui désigne SUSAN est en accord avec le rôle de Cp que ce référent obtient à l'issue de (19a), sa forme de reprise dans (19b') est difficilement compatible avec son statut de l'entité la plus saillante au moment où (19b') est énoncé, les noms propres étant utilisés soit pour introduire des référents nouveaux, i.e. ayant un degré d'accessibilité nul, soit pour réintroduire des référents anciens pour le discours mais qui n'occupent plus le devant de la scène discursive, i.e. ayant un degré d'accessibilité bas.

Si la théorie du centrage d'attention s'intéresse aux principes régissant la pronominalisation « locale » de référents présents dans des segments discursifs pluri-référentiels, le mécanisme qu'elle propose ne pouvant s'appliquer qu'à deux énoncés juxtaposés au sein d'un discours, la SDRT aborde ce phénomène d'un point de vue plus « global » en ce sens qu'elle essaie de rendre compte de la pronominalisation non adjacente, i.e. des cas où les pronoms clitiques présents dans un énoncé $\mathrm{E}_{\mathrm{n}}$ ne se rapportent pas à des référents mentionnés par $\mathrm{E}_{\mathrm{n}-1}$ mais à ceux dont il a été question auparavant, i.e. dans $\mathrm{E}_{\mathrm{n}-2}$, par exemple. Afin d'expliquer les contraintes qui pèsent sur l'emploi de ce type d'anaphores pronominales non adjacentes, qui semblent ne pas être admises dans tous les contextes (cf. (20) vs (21) ci-dessous), Asher \& Lascarides (1998) postulent que la relation rhétorique par laquelle un constituant $\beta$ est lié à un constituant précédent $\alpha$ détermine non seulement la disponibilité de $\alpha$ en tant que site d'attachement discursif pour un constituant $\gamma$ suivant $\beta$ (cf. supra), mais également l'aptitude de $\alpha$ à comporter l'antécédent d'un pronom clitique contenu dans $\gamma$.

Plus concrètement, si, dans une séquence $(\alpha, \beta, \gamma), \beta$ est coordonné à $\alpha$, alors seules les entités mentionnées par $\beta$ seront des antécédents possibles d'une proforme figurant dans $\gamma$, comme c'est le cas dans (20) ci-dessous. En effet, le fait que (b) est coordonné à (a) via Narration empêche (c) d'entrer en relation discursive avec (a) de même que d'utiliser le référent présent dans (a) mais absent dans (b), i.e. CAMBRIOLEUR, comme antécédent du pronom sujet $i l$. Si, en revanche, $\beta$ est subordonné à $\alpha$, alors une proforme apparaissant dans $\gamma$ peut se référer soit aux individus introduits par $\alpha$, soit à ceux évoqués par $\beta$. Crucialement, à cet égard, comme l'illustre (21) ci-dessous, la relation d'Arrière-plan, à la différence de celle de Narration, se comporte comme une relation de subordination en ce que si $\beta$ est relié à $\alpha$ par Arrière-plan, $\alpha$ reste accessible pour le discours qui suit $\beta$ tant rhétoriquement que référentiellement : 
(20) a. Un cambrioleur s'est introduit dans l'appartement de Marie.

b. Elle s'en est rendu compte le lendemain.

c. ? Il a emporté tous ses bijoux.

(21) a. Un cambrioleur s'est introduit dans l'appartement de Marie.

b. Elle dormait.

c. Il a emporté tous ses bijoux.

Partant de là, on peut se demander de quelle manière des relatives appositives comme celles présentées dans (22a) et (22b) affectent l'accessibilité des référents figurant dans leur segment discursif d'accueil et si leur effet est comparable à celui produit par les propositions indépendantes du même contenu :

(22) a. [Louise fit signe au garçon $]_{\mathrm{E} 1}$, [qui quitta à contrecœur son tabouret $]_{\mathrm{E} 2}$.

b. [Louise fit signe au garçon] $]_{\mathrm{E} 1}$, [qui parlait au téléphone en feuilletant distraitement un journal de mode posé sur le comptoir] $]_{\mathrm{E} 2}$.

(23) a. [Louise fit signe au garçon $]_{\mathrm{E} 1}$. [Il quitta à contrecœur son tabouret $]_{\mathrm{E} 2}$.

b. [Louise fit signe au garçon] $]_{\mathrm{E} 1}$. [Il parlait au téléphone en feuilletant distraitement un journal de mode $]_{\mathrm{E} 2}$.

$\mathrm{Si}$, comme le postule Holler, les relatives appositives sont équivalentes discursivement aux propositions indépendantes, les deux types de structures réalisant des unités du discours autonomes, alors on devrait s'attendre à ce qu'il n'y ait pas de différences significatives entre les prolongations auxquelles donnent lieu (22a) et (23a) d'une part et (22b) et (23b) d'autre part. Toutefois, compte tenu des contraintes sur la pronominalisation formulées par la SDRT, on s'attend à ce qu'il y ait une différence significative entre les séquences où $E_{2}$ est lié à $E_{1}$ par Narration et celles où $E_{2}$ est lié à $E_{1}$ par Arrière-plan. Dans le premier cas, les pronoms clitiques contenus dans $\mathrm{E}_{3}$ devraient renvoyer massivement au $\mathrm{Cp} \mathrm{d}^{\prime} \mathrm{E}_{2}$, i.e. GARÇON; et, dans le second, ils pourraient se rapporter aussi bien au $\mathrm{Cp} \mathrm{d}^{\prime} \mathrm{E}_{1}$, i.e. LOUISE, qu'à celui d' $\mathrm{E}_{2}$.

$\mathrm{Si}$, en revanche, comme nous le suggérons en nous fondant sur les données discutées dans la section précédente, les relatives appositives ne sont pas assimilables discursivement à leurs homologues indépendantes, on prédit que seules les séquences où $\mathrm{E}_{2}$ est réalisé par une proposition autonome, i.e. (23a) et (23b), seront sensibles à la structure rhétorique de leurs discours d'accueil. Pour ce qui est des relatives appositives, quelle que soit la relation qu'elles entretiennent avec leur matrice, seule cette dernière devrait être prise en compte lors du calcul du $\mathrm{Cr}$ le plus attendu de la suite immédiate. Autrement dit, les pronoms clitiques contenus dans $E_{3}$ suivant (22a) et (22b) devraient être majoritairement coréférentiels avec le $\mathrm{Cp}$ d' $\mathrm{E}_{1}$.

\section{Etude expérimentale du fonctionnement discursif des relatives narratives et descriptives}

Afin de tester ces prédictions, nous avons recouru à une méthodologie expérimentale en mettant en place un test de continuation? .

\subsection{Matériel expérimental}

Le matériel expérimental utilisé dans cette étude est constitué de cinq histoires du type de $(24)^{10}$ :

(24) Nicolas Brunois ${ }_{1}$, serveur dans un bar à Montmartre, prit une pause de 10 minutes. Il attrapa un paquet de cigarettes et enfila à la hâte sa parka. Comme il passait à côté du comptoir, il salua [un vieil homme vêtu d'une soutane $]_{2}, \%$.

A. qui agitait des brochures religieuses en toussant.

B. qui agita des brochures religieuses en toussant.

C. $\mathrm{Il}_{2}$ agitait des brochures religieuses en toussant.

D. $\mathrm{Il}_{2}$ agita des brochures religieuses en toussant. 
E. -

La partie invariable de chaque texte met en place deux référents humains - NICOLAS et VIEIL HOMME (ciaprès Réf 1 et Réf 2 respectivement), qui n'ont pas le même statut discursif. Le Réf 1 , repris régulièrement comme sujet de phrase réalisé par un pronom, maintient, tout au long du texte, son rôle de $\mathrm{Cr} / \mathrm{Cp}$. Le Réf 2 , en revanche, n'est ni $\mathrm{Cp}$, ni $\mathrm{Cr}$ de l'énoncé qui l'évoque pour la première fois, celui-ci, tout comme le discours précédent, étant centré sur le Réf 1 . En d'autres termes, l'état du focus d'attention résultant du traitement de la partie invariable de (24) laisse entendre que le Réf 1 gardera son statut de $\mathrm{Cr}$ dans l'énoncé subséquent.

Cette partie invariable est suivie de cinq conditions expérimentales, qui se distinguent selon deux critères : syntaxique (indépendante $v s$ subordonnée) et discursif (narration $v s$ arrière-plan). Ainsi, la condition $\mathrm{A}$ correspond à une relative d'arrière-plan (ci-après descriptive), $\mathrm{B}$ à une relative narrative, $\mathrm{C}$ à une indépendante descriptive et $\mathrm{D}$ à une indépendante narrative. Dans les conditions [+ relative], il s'agit toujours de relatives à sujet relativisé, i.e. en qui, qui ont l'avantage de donner lieu à des phrases complexes projetant un Cp différent (le Réf 1 ou le Réf 2) suivant qu'on les traite comme constituées d'une seule ou de deux unités discursives différentes. La cinquième condition, E, qui ne comporte aucune suite portant sur le Réf 2 (ni sur le Réf 1), est une condition contrôle que nous avons intégrée pour nous assurer que les effets observés dans les autres configurations ( $\mathrm{s}$ 'il y en a) peuvent être attribués à elles seules et ne sont pas dus à des facteurs indépendants.

\subsection{Procédure}

Les cinq textes expérimentaux, "mélangés » avec quatre distracteurs, ont été répartis en cinq livrets, conçus en sorte qu'une histoire n'apparaisse que sous une des cinq variantes possibles (A, B, C, D ou E). Ainsi, suivant le livret dans lequel elle apparait, l'histoire citée dans (24) figure sous une des variantes présentées dans $(25)-(29)$ :

(25) A. Nicolas Brunois1, serveur dans un bar à Montmartre, prit une pause de 10 minutes. [...] il salua un vieil homme vêtu d'une soutane, qui agitait des brochures religieuses en toussant.

(26) B. Nicolas Brunois1, serveur dans un bar à Montmartre, prit une pause de 10 minutes. [...] il salua un vieil homme vêtu d'une soutane, qui agita des brochures religieuses en toussant.

(27) C. Nicolas Brunois1, serveur dans un bar à Montmartre, prit une pause de 10 minutes. [...] il salua un vieil homme vêtu d'une soutane. Il agitait des brochures religieuses en toussant.

(28) D. Nicolas Brunois1, serveur dans un bar à Montmartre, prit une pause de 10 minutes. [...] il salua un vieil homme vêtu d'une soutane. Il agita des brochures religieuses en toussant.

(29) E. Nicolas Brunois1, serveur dans un bar à Montmartre, prit une pause de 10 minutes. [...] il salua un vieil homme vêtu d'une soutane. $\underline{\varnothing}$

50 participants, étudiants en linguistique à l'Université Sorbonne Nouvelle - Paris 3, ont reçu la tâche suivante: Les textes qui suivent sont des débuts de romans. Imaginez deux phrases prolongeant directement le début de l'histoire.

\subsection{Prédictions sur les continuations attendues}

Comme nous l'avions évoqué ci-dessus, en suivant l'hypothèse d'indépendance discursive des relatives appositives, on prédit qu'il n'y aura pas de différences significatives entre B [+ relative narrative] et D [+ indépendante narrative] d'une part et entre $\mathrm{A}$ [+ relative descriptive] et $\mathrm{C}$ [+ indépendante descriptive] d'autre part, le premier groupe réservant massivement le rôle de Cr au Réf 2 (30) et le second admettant aussi bien la pronominalisation du Réf 1 que celle du Réf 2 (31) :

(30) $[\ldots] \mathrm{il}_{1}[=$ Nicolas $]$ salua [un vieil homme vêtu d'une soutane $]_{2} /$, Il/qui agita des brochures religieuses en toussant.

a. Il fixait Nicolas d'un regard plein de réprobation. / $\mathrm{Il}_{2}$ le fixait d'un regard plein de réprobation. $\mathrm{OU}$

b. Nicolas lui proposa un verre d'eau. OU 
c. Quelques minutes plus tard, $\mathrm{il}_{2}$ rangea les brochures et quitta le bar. OU

d. Sans s'arrêter, Nicolas passa à la terrasse.

(31) $[\ldots] \mathrm{il}_{1}[=\text { Réf 1] salua [un vieil homme vêtu d'une soutane }]_{2} . /$, Il/qui agitait des brochures religieuses en toussant.

a. Il proposa un verre d'eau au vieil homme. / $\mathrm{Il}_{1}$ lui 2 proposa un verre d'eau. OU

b. Il fixait Nicolas d'un regard plein de réprobation. / $\mathrm{Il}_{2} \mathrm{le}_{1}$ fixait d'un regard plein de réprobation. OU

c. Sans s'arrêter, $\mathrm{il}_{1}$ passa à la terrasse. OU

d. Quelques minutes plus tard, $\mathrm{il}_{2}$ rangea les brochures et quitta le bar.

Pour ce qui est de l'hypothèse de dépendance discursive des relatives appositives que nous avançons, les prédictions auxquelles elle donne lieu se présentent comme suit. Il devrait y avoir une différence significative entre les prolongations écrites après de $\mathrm{A}$ et $\mathrm{B}$ d'une part et de $\mathrm{C}$ et $\mathrm{D}$ d'autre part. On s'attend à ce que dans le premier cas (i.e. A et $\mathrm{B}$ ), seul le $\mathrm{Cp}$ de la matrice - le Réf 1 - soit projeté comme le $\mathrm{Cr}$ le plus attendu de la suite et donc les reprises pronominales soient majoritairement réservées au Réf 1 , comme cela est illustré dans (32):

(32) $[\ldots] \mathrm{il}_{1}[=\text { Nicolas] salua [un vieil homme vêtu d'une soutane }]_{2}$, qui agitait/agita des brochures religieuses en toussant.

a. $\mathrm{Il}_{1} \mathrm{lui}_{2}$ proposa un verre d'eau. / Il proposa un verre d'eau au vieil homme. OU

b. Le vieil homme lui tendit une brochure. OU

c. Sans s'arrêter, $\mathrm{il}_{1}$ passa à la terrasse. $\mathrm{OU}$

d. Quelques minutes plus tard, le vieil homme rangea les brochures et quitta le bar.

La distribution des proformes clitiques dans $\mathrm{C}$ et $\mathrm{D}$ devrait, en revanche, être sensible au facteur rhétorique, i.e. suivre le modèle exemplifié dans (30) et (31) ci-dessus. Les continuations engendrées par la condition contrôle E devraient être du type de (32) et cela indépendamment de l'hypothèse sur le statut discursif des relatives appositives à l'examen.

\subsection{Traitement du corpus}

Le corpus réuni, qui contient $45^{11}$ prolongations écrites dans la condition $\mathrm{A}, 47$ dans la condition $\mathrm{B}, 46$ dans la condition $\mathrm{C}, 45$ dans $\mathrm{D}$ et 48 textes rédigés dans $\mathrm{E}$, a été annoté selon les critères suivants : (a) prolongations où le Réf 1 est le $\mathrm{Cp} / \mathrm{Cr}$ (33a); (b) prolongations où le Réf 2 est le $\mathrm{Cp} / \mathrm{Cr}$ (33b); (c) prolongations où le Réf 1 est réalisé par une expression autre qu'un pronom il ou elle (33c) ; (d) prolongations où le Réf 2 est le seul élément pronominalisé, i.e. où il est le Cr sans être forcément le Cp, (33c) ou $(33 d)$ :

(33) [...] ill [= Nicolas] salua [un vieil homme vêtu d'une soutane]2./, Il/qui agita/agitait des brochures religieuses en toussant.

a. Il proposa un verre d'eau au vieil homme. / $\mathrm{Il}_{1}$ lui proposa un verre d'eau.

b. Il fixait Nicolas d'un regard plein de réprobation. / $\mathrm{Il}_{2}$ le fixait d'un regard plein de réprobation.

c. Nicolas / Le barman lui proposa un verre d'eau.

d. Il tendit une brochure à Nicolas.

Les données quantitatives obtenues ont été comparées par un test de Chi 2.

\subsection{Résultats}

\section{(a) prolongations où le rôle de $\mathrm{Cp} / \mathrm{Cr}$ est réservé au Réf 1}

(34) $[\ldots] \mathrm{il}_{1}$ salua [un vieil homme vêtu d'une soutane $]_{2}$, . [A/B/C/D/E]. Sans s'arrêter, $\mathrm{il}_{1}$ passa sur la terrasse. 


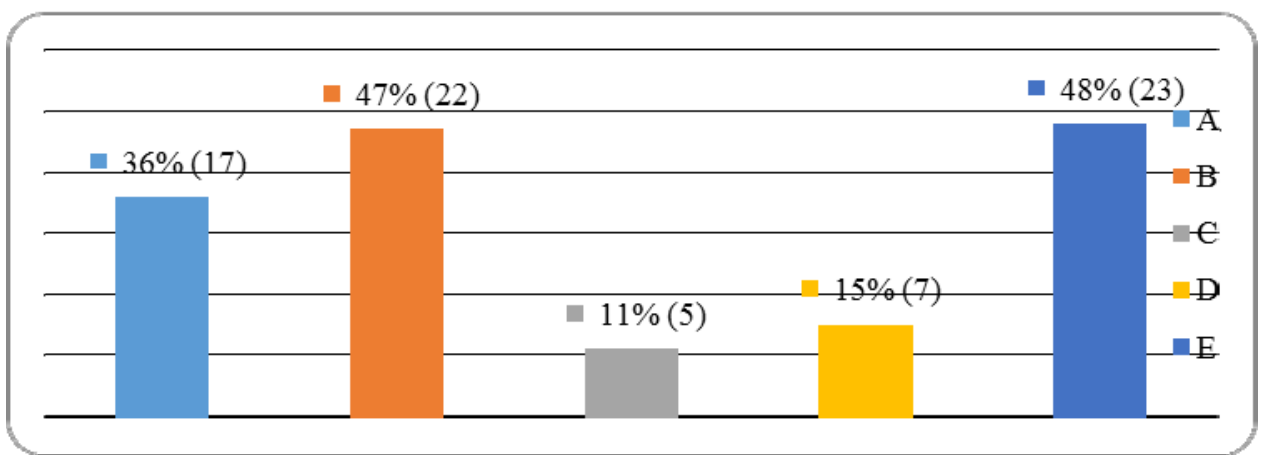

Graphique 1 - Prolongations avec le Réf 1 en tant que $\mathrm{Cp} / \mathrm{Cr}$

Les différences sont hautement significatives entre les conditions A, B [+ relative] et E [Ø] d'une part et les conditions $\mathrm{C}$ et $\mathrm{D}[+$ indépendante] d'autre part $(\mathrm{p}=0,000007)$, le premier groupe donnant lieu à un nombre plus important de prolongations où le Réf 1 est réalisé comme $\mathrm{Cp} / \mathrm{Cr}$. Les différences observées à l'intérieur de chaque groupe syntaxique, A \& B : [+relative] et C \& D : [+indépendante], ne sont pas significatives.

\section{(b) prolongations où le rôle de $\mathrm{Cp} / \mathrm{Cr}$ est réservé au Réf $\mathbf{2}$}

(35) $[\ldots]$ il $1_{1}$ salua [un vieil homme vêtu d'une soutane $]_{2},$. [ $[\mathrm{A} / \mathrm{B} / \mathrm{C} / \mathrm{D} / \mathrm{E}] . \mathrm{Il}_{2}$ se mit à rire en voyant Nicolas.

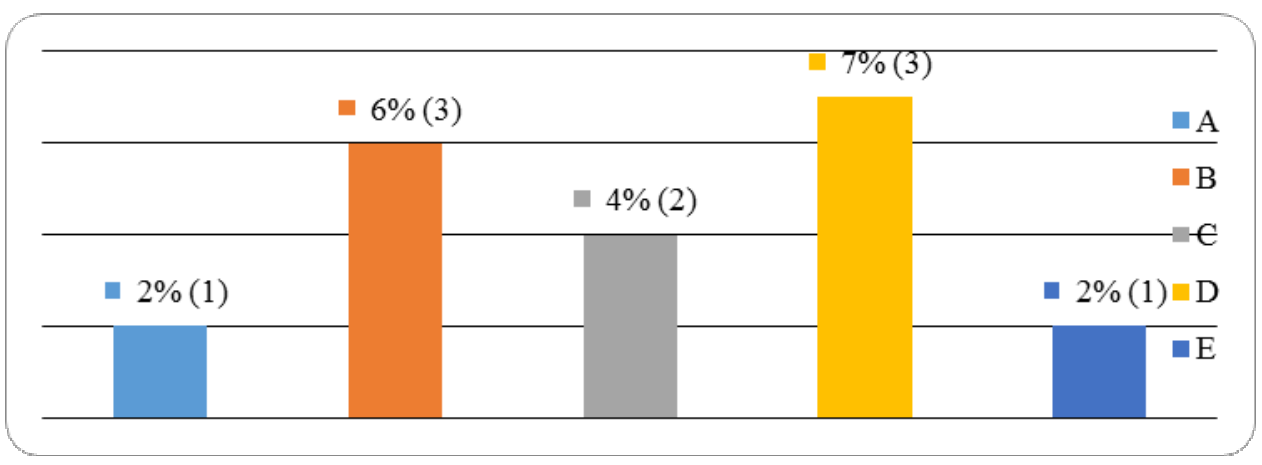

Graphique 2 - Prolongations avec le Réf 2 en tant que $\mathrm{Cp} / \mathrm{Cr}$

Etant donné que les continuations de ce type sont extrêmement rares et cela quelle que soit la condition examinée (A, B, C, D ou E), les différences observées dans le tableau 2 ne peuvent pas être vérifiées statistiquement ${ }^{12}$.

\section{(c) prolongations où le Réf 1 est désigné par une expression autre qu'un pronom clitique}

(36) a. $[\ldots] \mathrm{il}_{1}$ salua [un vieil homme vêtu d'une soutane $]_{2}, / .[\mathrm{A} / \mathrm{B} / \mathrm{C} / \mathrm{D} / \mathrm{E}]$. $\mathrm{Il}_{2}$ se mit à rire en voyant Nicolas. b. $[\ldots] \mathrm{il}_{1}$ salua [un vieil homme vêtu d'une soutane $]_{2}$, . [A/B/C/D/E]. Nicolas lui ${ }_{2}$ proposa un verre d'eau. 


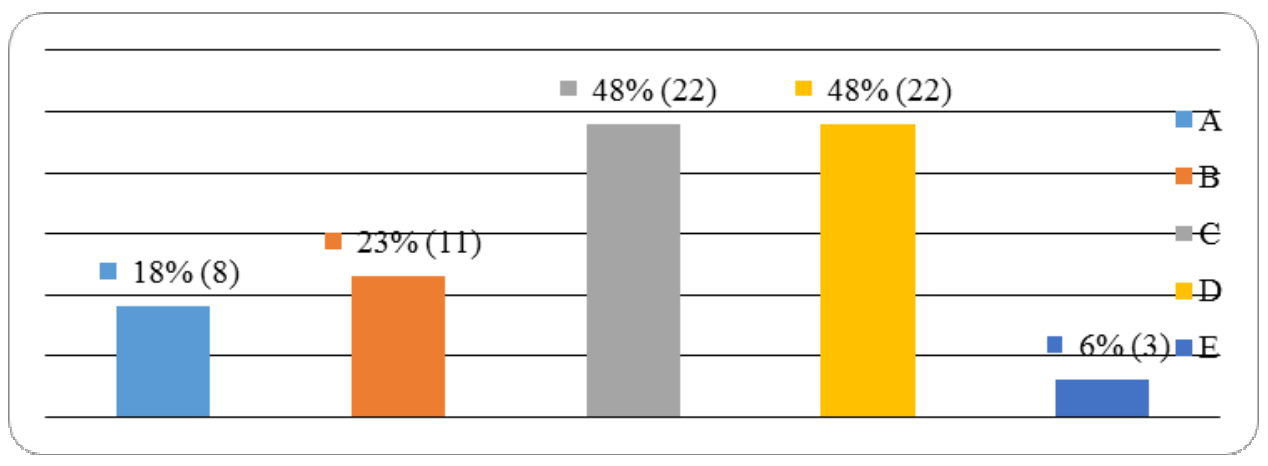

Graphique 3 - Prolongations où le Réf 1 est réalisé par une expression autre qu'un pronom clitique

Les différences sont hautement significatives entre le groupe A \& B d'une part et le groupe C \& D d'autre part $(p=0,00008)$. Autrement dit, les conditions qui réintroduisent le Réf 2 comme sujet d'une proposition indépendante donnent davantage de prolongations où le Réf 1 est évoqué par une expression autre qu'un pronom clitique.

\section{(d) prolongations où le Réf 2 est le seul élément pronominalisé}

(37) a. [...] il $1_{1}$ salua [un vieil homme vêtu d'une soutane $]_{2}$, . [A/B/C/D/E]. Nicolas lui $i_{2}$ tendit la main.

b. $[\ldots]$ il $1_{1}$ salua $[\text { un vieil homme vêtu d'une soutane }]_{2}, .[\mathrm{A} / \mathrm{B} / \mathrm{C} / \mathrm{D} / \mathrm{E}]$. $\mathrm{Il}_{2}$ demanda un verre d'eau à Nicolas.

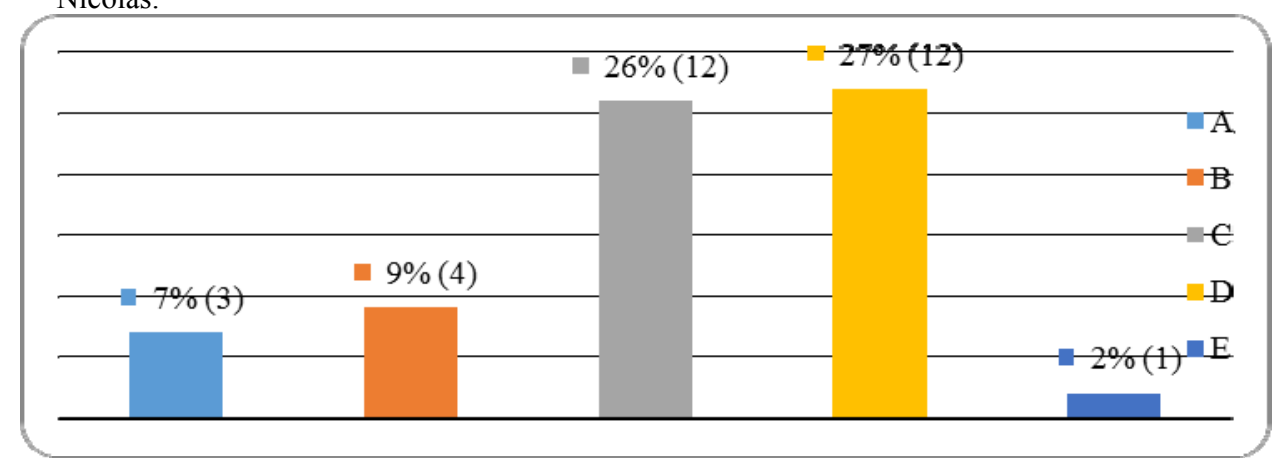

Graphique 4 - prolongations ou le Réf 2 est le seul élément pronominalisé

Encore une fois, les différences observables sont significatives entre les conditions A et B d'une part et les conditions $\mathrm{C}$ et $\mathrm{D}$ d'autre part $(\mathrm{p}=0,00009)$, le deuxième groupe donnant lieu à un nombre plus important de suites où le Réf 2 est le seul élément pronominalisé.

\subsection{Discussion}

Tout d'abord, il faut noter qu'aucune des cinq conditions étudiées ne permet au Réf 2 d'acquérir un degré d'accessibilité suffisamment élevé pour devenir le $\mathrm{Cr} / \mathrm{Cp}$ de la suite immédiate, dans la mesure où du point de vue du nombre de prolongations où il est retenu en cette qualité, $\mathrm{A}$ et $\mathrm{B}$, tout comme $\mathrm{C}$ et $\mathrm{D}$, sont à peine différenciables d'E (cf. graphique 2). Si l'on accepte la corrélation entre la notion de $C p$ de la théorie du centrage et celle, plus traditionnelle, de topique relationnel (Lambrecht, 1994), i.e. l'entité dont on parle et à laquelle se rapporte le commentaire qui suit, alors ce que suggèrent les données présentées dans le graphique 2 est que le Réf 2 , tout en ayant le potentiel de jouer le rôle de $\mathrm{Cr}$ (cf. graphique 4), est difficilement compatible avec la fonction de $\mathrm{Cp}$ ou, en d'autres termes, avec la fonction de topique relationnel du discours subséquent. En effet, dans toutes les conditions testées (A, B, C, D ou E), le Réf 2 tend à apparaître moins fréquemment comme sujet de la suite que le Réf 1 et cela quelle que soit sa forme de réalisation linguistique (i.e. pronominale ou non pronominale). Ainsi, alors que le Réf 1 figure comme sujet de la première phrase écrite en prolongement du matériel expérimentant dans $58 \%$ des cas, cette 
même fonction est assignée au Réf 2 dans $31 \%$ des cas seulement. A notre avis, cet état de choses (i.e. la difficulté de faire du Réf 2 le topique de la suite) est déclenché en partie par la forme même d'introduction des deux référents. A la différence du Réf 1, qui est importé dans le discours par un nom propre, le Réf 2 est évoqué pour la première fois par un GN indéfini uniquement. Or, selon Charolles (2002), bien que ces deux expressions soient utilisées pour introduire une entité dans l'univers du discours, elles n'assignent pas la même «importance» au référent qu'elles désignent. Un nom propre signale que son porteur va se trouver, très prochainement, au centre des préoccupations des interlocuteurs. Autrement dit, le référent désigné par un nom propre est, en quelque sorte, prédestiné à revenir régulièrement dans la suite du discours, i.e. à devenir son topique. Les GN indéfinis ne véhiculent pas cette instruction interprétative et donc sont employés plus fréquemment pour mentionner des individus secondaires, réservés à jouer un rôle épisodique dans le récit.

Quant aux données présentées dans les graphiques 2, 3 et 4, elles vont dans le même sens que celles qui reflètent les particularités de l'interprétation des relatives appositives vis-à-vis de l'opposition avant-plan $v s$ arrière-plan (cf. section 2) et invitent à conclure que du point de vue de leurs propriétés discursives, les relatives appositives, qu'elles soient narratives ou descriptives, ne peuvent pas être assimilées à leurs homologues indépendantes et que, par conséquent, la meilleure façon de rendre compte de leur comportement dans le discours est de les traiter comme des constituants dépourvus d'autonomie discursive, i.e. comme des constituants qui n'ont d'existence qu'en tant que partie intégrante de l'unité informative réalisée par leur proposition enchâssante.

En effet, toutes les différences quantitatives observées entre A, B, C et D sont toujours significatives entre les conditions qui se distinguent par le critère syntaxique, i.e. entre le groupe formé par les relatives (A et $\mathrm{B})$ et celui formé par les indépendantes $(\mathrm{C}$ et $\mathrm{D})$. Ainsi, contrairement à ces dernières, $\mathrm{A}$ et $\mathrm{B}$ rejoignent $\mathrm{E}$ du point de vue du nombre de prolongations où le Réf 1 est retenu comme $\mathrm{Cp} / \mathrm{Cr}$ (cf. graphique 1 ) et où le Réf 2 apparaît comme le seul élément pronominalisé (cf. graphique 4). Et, bien que, comparées à E, elles tendent à employer plus souvent une expression nominale pleine pour réintroduire le Réf 1 (cf. graphique 3 ), les chiffres qu'elles donnent sont significativement inférieurs à ceux attestés dans $\mathrm{C}$ et $\mathrm{D}$. Or, ces faits posent problème à toute approche qui traite les relatives appositives comme des unités du discours autonomes, qu'elle s'inscrive dans le cadre de la théorie du centrage d'attention ou celui de la SDRT. Si les phrases complexes constituées d'une matrice et d'une relative appositive interviennent séparément dans leur discours d'accueil comme le feraient deux propositions indépendantes du même contenu, ce qui reste inexplicable du point de vue de la théorie du centrage c'est pourquoi après une relative appositive, les deux référents - le Réf 1 et le Réf 2 - maintiennent quasiment intacts les statuts qu'ils ont acquis à l'issue du discours précédent (i.e. central et non central respectivement), et après une proposition indépendante le Réf 2 parvient à obtenir un degré de saillance suffisamment élevé pour rivaliser avec le Réf 1 au rôle de Cr de l'énoncé subséquent. En effet, $\mathrm{C}$ et $\mathrm{D}$ diminuent considérablement la fréquence de prolongations où le Réf 1 est élu comme $\mathrm{Cp} / \mathrm{Cr}$ et augmentent le nombre de suites où il est désigné par une expression nominale pleine. Cela signifie qu'à la différence de ce qui se passe dans $\mathrm{A}$ et $\mathrm{B}$, dans $\mathrm{C}$ et $\mathrm{B}$, le degré d'accessibilité du Réf 1 baisse et cela grâce à l'émergence d'un nouveau candidat possible au statut de $\mathrm{Cr}$ de la suite, i.e. le Réf 2. Notons au passage que les résultats auxquels donnent lieu les conditions $\mathrm{A}, \mathrm{C}$ et $\mathrm{D}$ pris isolément sont tout à fait compatibles avec une analyse fondée sur la SDRT comme celle d'Holler. En effet, dans A et C, les propositions tests sont liées par Arrière-plan au discours antérieur, ce qui veut dire que dans ces cas de figure, les anaphores clitiques apparaissant dans la suite immédiate peuvent trouver leur antécédent aussi bien dans le contenu des propositions tests que dans celui des propositions auxquelles elles s'attachent. Il en va de même pour la condition D : étant donné qu'ici, la proposition test est liée à ce qui précède par Narration, il est tout à fait attendu que seuls les référents évoqués dans ladite proposition soient susceptibles d'être repris par un pronom clitique. Toutefois, ce qui reste à expliquer c'est d'une part pourquoi $\mathrm{A}$ et $\mathrm{C}$ affichent des tendances opposées, la première favorisant davantage que la seconde la cliticisation du Réf 1 et, d'autre part, pourquoi $\mathrm{B}$, sur ce point, rejoint $\mathrm{C}$ et non pas $\mathrm{D}$, alors que les deux (B et $\mathrm{D})$ sont reliées par Narration à leur discours précédent. 
L'hypothèse de dépendance discursive des relatives appositives nous semble, par conséquent, plus motivée empiriquement. Premièrement, elle explique assez facilement pourquoi c'est le Réf 1 qui se voit massivement assigner le statut de $\mathrm{Cr}$ dans les prolongations écrites dans $\mathrm{A}$ et $\mathrm{B}$, autrement dit, pourquoi il maintient un degré d'accessibilité maximal malgré l'intervention d'une relative appositive centrée exclusivement sur le Réf 2. En effet, étant donné que le calcul du $\mathrm{Cr}$ de l'énoncé (i.e. d'une unité informative autonome) $\mathrm{E}_{\mathrm{n}}$ se fait à partir du $\mathrm{Cp}$ de l'énoncé $\mathrm{E}_{\mathrm{n}-1}$, le fait que les relatives appositives ne constituent d'énoncés implique automatiquement qu'il n'y a qu'un seul Cp par phrase complexe avec une relative appositive - le référent sujet de la prédication principale, i.e. le Réf 1. Deuxièmement, traiter les relatives appositives comme étant intégrées dans l'unité informative réalisée par leur matrice pourrait également expliquer pourquoi les effets que les relations rhétoriques de Narration et d'Arrière-plan reliant $\alpha$ et $\beta$ produisent dans le discours se manifestent si $\alpha$ et $\beta$ sont exprimés par des structures indépendantes et disparaissent si $\beta$ correspond à une relative appositive : si les relatives appositives et leurs homologues indépendantes ont des statuts discursifs différents, il est tout à fait logique de s'attendre à ce qu'elles ne soient pas sujettes aux mêmes règles et contraintes discursives et qu'elles aient, de $\mathrm{c}$ fait, des fonctionnements discursifs divergents.

\section{Conclusions}

Dans le présent travail nous nous sommes proposé de tester empiriquement l'hypothèse d'indépendance discursive des subordonnées relatives appositives, formulée par Brandt (1900), Lambrecht (1998), Holler (2005b) inter alia, et, corollairement, le bien-fondé de la distinction fonctionnelle, régulièrement mentionnée dans la littérature sur l'organisation textuelle, entre les relatives dites narratives et descriptives. Pour ce faire, nous avons recouru à une méthodologie expérimentale en mettant en place un test de continuation. L'objectif du test était de voir à quel point sont empiriquement justifiées les prédictions concernant l'effet des relatives appositives sur l'accessibilité des référents présents dans leur segment discursif d'accueil, prédictions auxquelles donnent lieu les analyses assimilant discursivement les phrases complexes avec une relative appositive à des séquences de deux propositions indépendantes juxtaposées.

Les résultats issus de cette étude expérimentale nous suggèrent que, contrairement à ce que postule la littérature antérieure, les relatives appositives ne peuvent pas être assimilées discursivement aux prédications autonomes, car, malgré le lien sémantico-syntaxique relâché qu'elles semblent entretenir avec leur matrice, sur le plan du discours, elles sont intégrées en tant que constituant dépendant dans l'unité informative réalisée par leur proposition principale, et cela qu'il s'agisse de relatives narratives ou descriptives.

\section{ANNEXE}

Matériel utilisé dans l'étude expérimentale présentée ci-dessus :

Histoire 2 : Cécile Darnol, propriétaire d'une boutique de luxe, regarda attentivement la vitrine. Elle fit bouffer une jupe parapluie et rajouta un foulard en satin. Comme elle traversait le rayon « Accessoires en cuir », elle s'approcha d'une vieille femme mal habillée, /.

A. qui demandait de petites pièces en pleurant.

B. qui demanda de petites pièces en pleurant.

C. Elle demandait de petites pièces en pleurant.

D. Elle demanda de petites pièces en pleurant.

E. - 
Histoire 3 : Eloïse Duval, institutrice dans une école maternelle, franchit la grille de la cour de récréation. Elle traversa la rue et se dirigea vers un square. Comme elle s'installait sur un banc en plein soleil, elle fit signe à une petite fille aux cheveux roux, $/$.

A. qui faisait des bulles de savon en riant.

B. qui fit des bulles de savon en riant.

C. Elle faisait des bulles de savon en riant.

D. Elle fit des bulles de savon en riant.

E. -

Histoire 4 : François Levestre, laveur de carreaux à la Tour Montparnasse, descendit d'un étage. Il vérifia les fixations de la nacelle et mit des gants en caoutchouc. Comme il s'attaquait à une baie vitrée coulissante, il fit signe de la main à un homme d'affaires assis derrière un bureau, /.

A. qui rangeait des papiers en chantonnant.

B. qui rangea des papiers en chantonnant.

C. Il rangeait des papiers en chantonnant.

D. Il rangea des papiers en chantonnant.

E. -

Histoire 5 : Patrick Landois, greffier au tribunal correctionnel de Paris, s'approcha de la salle d'audience. Il rectifia le nœud de sa cravate et poussa la porte. Comme il remontait les bancs à moitié vides, il arriva près d'un jeune homme couvert d'ecchymoses, /.

A. qui poussait des cris de douleur en gémissant.

B. qui poussa des cris de douleur en gémissant.

C. Il poussait des cris de douleur en gémissant.

D. Il poussa des cris de douleur en gémissant.

E. -

\section{Références bibliographiques}

Ariel, M. (1990). Accessing Noun-Phrase Antecedents. Londres : Routledge.

Asher, N. (1993). Reference to abstract objects in discourse. Dordrecht : Kluwer.

Asher, N. \& Lascarides, A. (1993). Temporal Interpretation, Discourse Relations and Commonsense Entailment. Linguistics and Philosophy 16, 437-493.

Asher, N. \& Lascarides, A.1(998). Semantics and Pragmatics of presupposition. Journal of Semantics 15/3, 239-300.

Asher, N. \& Vieu, L. (2005). Subordinating and coordinating discourse relations. Lingua 115/4, 591-610.

Brandt, M. (1990). Weiterführende Nebensätze Zu ihrer Syntax, Semantik und Pragmatik, Stockholm : Almquist \& Wiksell.

Charolles, M. (2002). La référence et les expressions référentielles en français. Paris : Ophrys.

Charolles, M. (2007). Comment évaluer les effets des relatives en qui sur les chaînes de coréférence. In Charolles, M. et al. (éd.), Parcours de la phrase - Mélanges offerts à Pierre Le Goffic, Paris : Ophrys, 193-212.

Combettes, B. (1992). L'organisation du texte. Metz : Centre d'Analyse Syntaxique de l'Université de Metz.

Cornilescu, A. (1996). Montague grammar and the analysis of relative clauses. București : Editura Universității din București. 
Cornish, F. (2000). L'accessibilité cognitive des référents, le centrage d'attention et la structure du discours : une vue d'ensemble. Verbum 22.1, 7-30.

Emonds, J. (1979). Appositive relatives have no properties. Linguistic Inquiry 10, 211-243.

Holler, A. (2005a). Weiterführende Relativsätze. Empirische und theoretische Aspekte. Berlin : Akademie Verlag.

Holler, A. (2005b). Expressing communicative-weight assignment discourse structurally. In Proceedings of the Workshop on Constraints in Discourse. Dortmund, 88-95.

Krifka, M. (2009). Embedding Speech Acts, ms. Humboldt Universität.

Lambrecht, K. (1994). Information structure and sentence form. Cambridge : Cambridge University Press.

Lambrecht, K. (1998). There was a farmer had a dog: syntactic amalgams revisited. In Axmaker, S. et al. (éd), Berkeley Linguistic Society Proceedings of the 14th Annual Meeting, Berkeley : UC Berkeley, CA, 319-339.

Loock, R. (2007). Appositive relative clauses and their functions in discourse. Journal of pragmatics 39.2, 336-362.

Peterson, P. (2004). Non-restrictive relatives and other non-syntagmatic relations in an LF framework. In Proceedings of LFG 2004, Stanford : CSLI Publications, 391-397.

Poesio, M. et al. (2004). Centering: a parametric theory and its instantiations. Computational Linguistics 30.3, 309363.

Reinhart, T. (1981). Pragmatics and linguistics: an analysis of sentence topics, Philosophica 27, 53-94.

Simons, M. et al. (2010). What projects and why. In Proceedings of SALT 20, 309-327.

Thompson, S. (1987). Subordination and narrative event structure. In Tomlin, R. (éd.), Coherence and grounding in discourse, Amsterdam : John Benjamins, 435-454.

Thorne, J. P. (1972). On nonrestrictive relative clauses. Linguistic Inquiry 3, 552-556.

Walker, M. (1998). Centering in naturally-occurring discourse: An overview. In Walker, M. et al. (éd.), Reference and referent accessibility, Amsterdam : John Benjamins, 291-306.

\footnotetext{
${ }^{1}$ Sur la corrélation entre les statuts syntaxique et discursif d'une proposition voir également Reinhart (1984), Thompson (1987).

${ }^{2}$ Tous les exemples de l'allemand sont tirés d'Holler (2005).

${ }^{3}$ Autrement dit, les relatives appositives possèdent une partition interne en topique / focus.

${ }^{4}$ La Théorie des représentations discursives segmentées (SDRT) 'Segmented Discourse Representation Theory' est une théorie de la cohérence et de la structure hiérarchique du discours.

5 Toutes les propriétés syntactico-sémantiques qui témoignent d'un lien extrêmement relâché que les relatives appositives entretiennent avec leurs matrices (cf. ci-dessus) s'appliquent uniformément aux relatives narratives et descriptives.

${ }^{6}$ Autrement dit, une séquence textuelle présentant une certaine homogénéité sémantique et pragmatique. Pour plus de détails, voir Cornish (2000).

${ }^{7}$ L'échelle de saillance/proéminence des Ca basée sur le critère syntaxique se présente comme suit : Sujet $>$ Objet indirect animé $>$ Objet direct $>$ Objet indirect inanimé $>$ Objet oblique (Walker \& al., 1998), où ' $\mathrm{x}>\mathrm{y}$ ' se lit comme
} 
' $x$ est plus saillant/proéminent que y'. Pour un aperçu d'autres facteurs susceptibles d'affecter la proéminence cognitive d'un référent, voir Cornish (2000), Poesio \& al., (2004).

${ }^{8}$ Les petites majuscules (SUSAN) marquent le référent du discours et les italiques (Susan) l'expression linguistique par laquelle ce référent est réalisé.

${ }^{9}$ Nous remercions M. Charolles de son aide dans l'élaboration et la réalisation de cette étude.

${ }^{10}$ La totalité du matériel expérimental utilisé dans cette étude est consultable dans l'annexe.

${ }^{11}$ Nous avons éliminé les continuations qui n'évoquent aucun des deux référents, comme par exemple, dehors, il pleuvait, etc.

${ }^{12}$ Les chiffres inférieurs à 5 ne peuvent pas subir le test que nous utilisons. Il en découle que dans la majorité des cas, la condition E ne peut pas être contrastée avec les autres conditions. 